\title{
Analytical Construction of Uniformly Convergent Method for Convection Diffusion Problem
}

\author{
Ali Filiz \\ Department of Mathematics \\ Faculty of Science \\ Pamukkale University \\ 20070 Denizli, Turkey \\ Email: alifiliz [AT] pau.edu.tr
}

\begin{abstract}
In this paper, we study the uniformly convergent method on equidistant meshes for the convectiondiffusion problem of type;
\end{abstract}

where $L^{*}$ the formal adjoint operator of $L$.

$$
L u=-\varepsilon u^{\prime \prime}+b u^{\prime}+c u=f(x), u(0)=0, \quad u(1)=0
$$

At the end of the this paper we will generate the scheme;

$$
-\frac{e^{\rho_{i}}}{e^{\rho_{i}}+1} U_{i-1}+U_{i}-\frac{1}{e^{\rho_{i}}+1} U_{i+1}=\left(f_{i}-c_{i} U_{i}\right) \frac{h}{b}\left(\frac{e^{\rho_{i}}-1}{e^{\rho_{i}}+1}\right)
$$

Keywords - Local Green's function, convection-diffusion problem, boundary value problem, singular points.

\section{INTRODUCTION}

We consider a uniformly convergent method, called Il'in-Allen-Southwell Scheme. First of all, we will show how to construct such a method. Then, numerical results can be done similar manner in future study.

\section{CONSTRUCTION OF UNIFORMLY CONVERGENT METHOD}

We describe a way of constructing a uniformly convergent difference scheme. We start with the standard derivation of an exact scheme for the convection-diffusion problem given above. The formal adjoint of $\mathrm{L}$ is defined by

$L^{*}=-\varepsilon \frac{d^{2}}{d x^{2}}-b \frac{d}{x}$

$L u=-\varepsilon u^{\prime \prime}+b u^{\prime}+c u=f(x), u(0)=0, u(1)=0$

Consider the following problem on the unit interval $\Omega=(0,1)$. Let $g_{i}$ be local Green's function of $L^{*}$ with respect to the point $x_{i}$; that is

$L^{*} g_{i}=-\varepsilon g_{i}^{\prime \prime}-b g_{i}^{\prime}=0$ in $\left(x_{i-1}, x_{i}\right) \cup\left(x_{i}, x_{i+1}\right)$

Let us impose boundary conditions

And impose additional conditions

$$
g_{i}\left(x_{i-1}\right)=g_{i}\left(x_{i+1}\right)=0
$$

$\varepsilon\left(g_{i}^{\prime}\left(x_{i}^{-}\right)-g_{i}^{\prime}\left(x_{i}^{+}\right)=1\right.$.

Now

$$
\begin{gathered}
\int_{x_{i-1}}^{x_{i+1}}(L u) g_{i} d x=\int_{x_{i-1}}^{x_{i+1}} f g_{i} d x \\
\int_{x_{i-1}}^{x_{i+1}}(L u) g_{i} d x=\int_{x_{i-1}}^{x_{i}}\left(-\varepsilon u^{\prime \prime}+b u^{\prime}\right) g_{i} d x+\int_{x_{i}}^{x_{i+1}}\left(-\varepsilon u^{\prime \prime}+b u^{\prime}\right) g_{i} d x
\end{gathered}
$$

Then apply integrating by parts 


$$
\begin{aligned}
\int_{x_{i-1}}^{x_{i}}\left(-\varepsilon u^{\prime \prime}+b u^{\prime}\right) & g_{i} d x+\int_{x_{i}}^{x_{i+1}}\left(-\varepsilon u^{\prime \prime}+b u^{\prime}\right) g_{i} d x \\
= & \left.\left(-\varepsilon u^{\prime}+b \mathrm{u}\right) g_{i}(x)\right|_{x_{i-1}} ^{x_{i}}-\int_{x_{i-1}}^{x_{i}}\left(-\varepsilon u^{\prime}+b \mathrm{u}\right) g_{i}^{\prime} d x+\left.\left(-\varepsilon u^{\prime}+b \mathrm{u}\right) g_{i}(x)\right|_{x_{i}} ^{x_{i+1}}-\int_{x_{i}}^{x_{i+1}}\left(-\varepsilon u^{\prime}+b \mathrm{u}\right) g_{i}^{\prime} d x \\
= & {\left[\left(-\varepsilon u^{\prime}\left(x_{i}^{-}\right)+b \mathrm{u}\left(x_{i}\right)\right) g_{i}\left(x_{i}\right)-\left(-\varepsilon u^{\prime}\left(x_{i-1}\right)+b \mathrm{u}\left(x_{i-1}\right)\right) g_{i}\left(x_{i-1}\right)\right] } \\
& +\left[\left(-\varepsilon u^{\prime}\left(x_{i+1}\right)+b \mathrm{u}\left(x_{i+1}\right)\right) g_{i}\left(x_{i+1}\right)-\left(-\varepsilon u^{\prime}\left(x_{i}^{+}\right)+b u\left(x_{i}\right)\right) g_{i}\left(x_{i}\right)\right] \\
& -\int_{x_{i-1}}^{x_{i}}(b \mathrm{u}) g_{i}^{\prime} d x-\int_{x_{i}}^{x_{i+1}}(b \mathrm{u}) g_{i}^{\prime} d x+\int_{x_{i-1}}^{x_{i}}\left(\varepsilon u^{\prime}\right) g_{i}^{\prime} d x+\int_{x_{i}}^{x_{i+1}}\left(\varepsilon u^{\prime}\right) g_{i}^{\prime} d x
\end{aligned}
$$

From properties of Green's function $\left(g_{i}\left(x_{i-1}\right)=g_{i}\left(x_{i+1}\right)=0\right)$ and $-\varepsilon g_{i}^{\prime \prime}-b g_{i}^{\prime}=0$ )

$$
\begin{gathered}
\int_{x_{i-1}}^{x_{i+1}}(L u) g_{i} d x=\int_{x_{i-1}}^{x_{i+1}} f g_{i} d x=-\varepsilon u^{\prime}\left(x_{i}^{-}\right) g_{i}\left(x_{i}\right)+\varepsilon u^{\prime}\left(x_{i}^{+}\right) g_{i}\left(x_{i}\right)+\left.(\varepsilon \mathrm{u}(\mathrm{x})) g_{i}(x)\right|_{x_{i-1}} ^{x_{i}}+\left.(\varepsilon \mathrm{u}(\mathrm{x})) g_{i}(x)\right|_{x_{i}} ^{x_{i+1}} \\
+\int_{x_{i-1}}^{x_{i}}\left(-\varepsilon g_{i}^{\prime \prime}-b g_{i}^{\prime}\right) \mathrm{u} d x+\int_{x_{i}}^{x_{i+1}}\left(-\varepsilon g_{i}^{\prime \prime}-b g_{i}^{\prime}\right) \mathrm{u} d x
\end{gathered}
$$

Since $u^{\prime}$ is continuous on $\left(x_{i-1}, x_{i+1}\right)$ then we have

$$
=\left[\varepsilon u\left(x_{i}\right) g_{i}^{\prime}\left(x_{i}^{-}\right)-\varepsilon u\left(x_{i-1}\right) g_{i}^{\prime}\left(x_{i-1}^{+}\right)\right]+\left[\varepsilon u\left(x_{i+1}\right) g_{i}^{\prime}\left(x_{i+1}^{-}\right)-\varepsilon u\left(x_{i}\right) g_{i}^{\prime}\left(x_{i}^{+}\right)\right]
$$

The identity can be written as

$$
-\varepsilon g_{i}^{\prime}\left(x_{i-1}\right) u_{i-1}+u_{i}+\varepsilon g_{i}^{\prime}\left(x_{i+1}\right) u_{i+1}=(f-c u) \int_{x_{i-1}}^{x_{i+1}} g_{i} d x
$$

General solution of second order differential equation (2) is given by

$-\varepsilon g_{i}^{\prime \prime}-b g_{i}^{\prime}=0$ is

$$
\begin{aligned}
& g_{i}\left(x_{i}^{-}\right)=c_{1}+c_{2}\left(-\frac{\varepsilon}{b}\right) e^{-\frac{b x}{\varepsilon}} \in\left(x_{i-1}, x_{i}\right) \\
& g_{i}\left(x_{i}^{+}\right)=c_{1}^{\prime}+c_{2}^{\prime}\left(-\frac{\varepsilon}{b}\right) e^{-\frac{b x}{\varepsilon}} \in\left(x_{i}, x_{i+1}\right)
\end{aligned}
$$

We have four unknowns $c_{1}, c_{2}, c_{1}^{\prime}$ and $c_{2}^{\prime}$, hence we need four equations:

$g_{i}\left(x_{i-1}\right)=0, \quad g_{i}\left(x_{i+1}\right)=0, \quad \varepsilon\left(g_{i}^{\prime}\left(x_{i}^{-}\right)-g_{i}^{\prime}\left(x_{i}^{+}\right)=1\right.$ and from continuity of $g_{i}$ at $x_{i}$ $g_{i}\left(x_{i}^{-}\right)=g_{i}\left(x_{i}^{+}\right)$

Imposing boundary conditions

$$
\begin{aligned}
& g_{i}\left(x_{i-1}\right)=c_{1}+c_{2}\left(-\frac{\varepsilon}{b}\right) e^{-\frac{b x_{i-1}}{\varepsilon}}=0 \\
& g_{i}\left(x_{i+1}\right)=c_{1}^{\prime}+c_{2}^{\prime}\left(-\frac{\varepsilon}{b}\right) e^{-\frac{b x_{i+1}}{\varepsilon}}=0
\end{aligned}
$$

By taking derivative of the equations (3) and (4)

$g_{i}^{\prime}\left(x_{i}^{-}\right)=c_{2}\left(-\frac{b}{\varepsilon}\right)\left(-\frac{\varepsilon}{b}\right) e^{-\frac{b x_{i-1}}{\varepsilon}}=c_{2} e^{-\frac{b x_{i}}{\varepsilon}}$

$g_{i}^{\prime}\left(x_{i}^{+}\right)=c_{2}^{\prime}\left(-\frac{b}{\varepsilon}\right)\left(-\frac{\varepsilon}{b}\right) e^{-\frac{b x_{i}}{\varepsilon}}=c_{2}^{\prime} e^{-\frac{b x_{i}}{\varepsilon}}$

Apply for the equation $\varepsilon\left(g_{i}^{\prime}\left(x_{i}^{-}\right)-g_{i}^{\prime}\left(x_{i}^{+}\right)=1\right.$ and get

$\varepsilon\left(c_{2} e^{-\frac{b x_{i}}{\varepsilon}}-c_{2}^{\prime} e^{-\frac{b x_{i}}{\varepsilon}}\right)=1 \Rightarrow c_{2}-c_{2}^{\prime}=\frac{1}{\varepsilon} e^{\frac{b x_{i}}{\varepsilon}}$

We have written $g_{i}\left(x_{i}^{-}\right)=g_{i}\left(x_{i}^{+}\right)$from continuity of $g_{i}$ at $x=x_{i}$

$g_{i}\left(x_{i}^{-}\right)-g_{i}\left(x_{i}^{+}\right)=0$

$c_{1}+c_{2}\left(-\frac{\varepsilon}{b}\right) e^{-\frac{b x_{i}}{\varepsilon}}-\left(c_{1}^{\prime}+c_{2}^{\prime}\left(-\frac{\varepsilon}{b}\right) e^{-\frac{b x_{i}}{\varepsilon}}\right)=0$

and then we have

$\left(c_{1}-c_{1}^{\prime}\right)+\left(c_{2}-c_{2}^{\prime}\right)\left(-\frac{\varepsilon}{b}\right) e^{-\frac{b x_{i}}{\varepsilon}}=0$

Let us simplify that

$\alpha_{i}=\frac{b x_{i}}{\varepsilon}$

$\rho_{i}=\frac{b h}{\varepsilon}$ 
We can write

$e^{\frac{b x_{i+1}}{\varepsilon}}=e^{\frac{b x_{i}+h}{\varepsilon}}=e^{\frac{b x_{i}}{\varepsilon}} \cdot e^{\frac{b h}{\varepsilon}}=e^{\alpha_{i}+\rho_{i}}$ and $e^{\frac{b x_{i-1}}{\varepsilon}}=e^{\frac{b x_{i}-h}{\varepsilon}}=e^{\frac{b x_{i}}{\varepsilon}} \cdot e^{-\frac{b h}{\varepsilon}}=e^{\alpha_{i}-\rho_{i}}$

We transform the equations

$c_{1}+c_{2}\left(-\frac{\varepsilon}{b}\right) e^{-\alpha_{i}+\rho_{i}}=0$

$c_{1}^{\prime}+c_{2}^{\prime}\left(-\frac{\varepsilon}{b}\right) e^{-\alpha_{i}-\rho_{i}}=0$

$c_{2}-c_{2}^{\prime}=\frac{1}{\varepsilon} e^{\alpha_{i}}$

$\left(c_{1}-c_{1}^{\prime}\right)+\left(c_{2}-c_{2}^{\prime}\right)\left(-\frac{\varepsilon}{b}\right) e^{-\alpha_{i}}=0$

Plug the equation (7) into the equation (8) we get

$\left(c_{1}-c_{1}^{\prime}\right)=\frac{1}{b}$

After using symbolic programming MATHEMATICA we obtain

$c_{1}=\frac{1}{b}\left(\frac{e^{\rho_{i}-1}}{e^{\rho_{i}-e^{-\rho_{i}}}}\right), \quad c_{2}=\frac{e^{\alpha_{i}}}{\varepsilon}\left(\frac{1-e^{-\rho_{i}}}{e^{\rho_{i}-e^{-\rho_{i}}}}\right)$

$c_{1}^{\prime}=\frac{1}{b}\left(\frac{e^{-\rho_{i-1}}}{e^{\rho_{i}}-e^{-\rho_{i}}}\right), \quad c_{2}^{\prime}=\frac{e^{\alpha_{i}}}{\varepsilon}\left(\frac{1-e^{\rho_{i}}}{e^{\rho_{i}}-e^{-\rho_{i}}}\right)$

Let us impose the equations (10) and (11), then we can rewrite the equations (3) and (4) as follows

$g_{i}\left(x_{i}^{-}\right)=c_{1}+c_{2}\left(-\frac{\varepsilon}{b}\right) e^{-\frac{b x}{\varepsilon}}=\frac{1}{b}\left(\frac{e^{\rho_{i-1}}}{e^{\rho_{i}-e^{-\rho_{i}}}}\right)+\frac{e^{\alpha_{i}}}{\varepsilon}\left(\frac{1-e^{-\rho_{i}}}{e^{\rho_{i}-e^{-\rho_{i}}}}\right)\left(-\frac{\varepsilon}{b}\right) e^{-\frac{b x}{\varepsilon}}$

$g_{i}\left(x_{i}^{+}\right)=c_{1}^{\prime}+c_{2}^{\prime}\left(-\frac{\varepsilon}{b}\right) e^{-\frac{b x}{\varepsilon}}=\frac{1}{b}\left(\frac{e^{-\rho_{i-1}}}{e^{\rho_{i}-e^{-\rho_{i}}}}\right)+\left(-\frac{\varepsilon}{b}\right) e^{-\frac{b x}{\varepsilon}} \frac{e^{\alpha_{i}}}{\varepsilon}\left(\frac{1-e^{\rho_{i}}}{e^{\rho_{i}-e^{-\rho_{i}}}}\right)$

Taking derivative equations (12) and (13),

$$
\begin{aligned}
& g_{i}^{\prime}\left(x_{i}^{-}\right)=\frac{1}{\varepsilon}\left(\frac{1-e^{-\rho_{i}}}{e^{\rho_{i}}-e^{-\rho_{i}}}\right) \\
& g_{i}^{\prime}\left(x_{i}^{+}\right)=\frac{1}{\varepsilon}\left(\frac{1-e^{\rho_{i}}}{e^{\rho_{i}}-e^{-\rho_{i}}}\right)
\end{aligned}
$$

where $\alpha_{i}=\frac{b x_{i}}{\varepsilon}$ and $\rho_{i}=\frac{b h}{\varepsilon}$. We can easily obtain

Now, we can calculate the integral using $g_{i}^{-}$and $g_{i}^{+}$

$$
\begin{aligned}
& g_{i}^{\prime}\left(x_{i-1}^{-}\right)=\frac{1}{\varepsilon}\left(\frac{e^{\rho_{i}}-1}{e^{\rho_{i}}-e^{-\rho_{i}}}\right) \\
& g_{i}^{\prime}\left(x_{i+1}^{-}\right)=\frac{1}{\varepsilon}\left(\frac{e^{-\rho_{i}}-1}{e^{\rho_{i}}-e^{-\rho_{i}}}\right)
\end{aligned}
$$

$$
\begin{gathered}
(f-c u) \int_{x_{i-1}}^{x_{i+1}} g_{i} d x=(f-c u)\left(\int_{x_{i-1}}^{x_{i}} g_{i}^{-} d x+\int_{x_{i}}^{x_{i+1}} g_{i}^{+} d x\right) \\
=(f-c u)\left(\int_{x_{i-1}}^{x_{i}}\left(\frac{1}{b}\left(\frac{e^{\rho_{i}}-1}{e^{\rho_{i}}-e^{-\rho_{i}}}\right)+\frac{e^{\alpha_{i}}}{\varepsilon}\left(\frac{1-e^{-\rho_{i}}}{e^{\rho_{i}}-e^{-\rho_{i}}}\right)\left(-\frac{\varepsilon}{b}\right) e^{-\frac{b x}{\varepsilon}}\right) d x\right. \\
\left.+\int_{x_{i}}^{x_{i+1}}\left(\frac{1}{b}\left(\frac{e^{-\rho_{i}}-1}{e^{\rho_{i}}-e^{-\rho_{i}}}\right)+\frac{e^{\alpha_{i}}}{\varepsilon}\left(\frac{1-e^{\rho_{i}}}{e^{\rho_{i}}-e^{-\rho_{i}}}\right)\left(-\frac{\varepsilon}{b}\right) e^{-\frac{b x}{\varepsilon}}\right) d x\right)
\end{gathered}
$$

İntegrate with respect to $x$;

$$
\begin{aligned}
=(f & -c u)\left[\left.\frac{1}{b}\left(\frac{e^{\rho_{i-1}}}{e^{\rho_{i}-e^{-\rho_{i}}}}\right) \cdot x\right|_{x_{i-1}} ^{x_{i}}+\left.\frac{e^{\alpha_{i}}}{\varepsilon}\left(\frac{1-e^{-\rho_{i}}}{e^{\rho_{i}-e^{-\rho_{i}}}}\right)\left(-\frac{\varepsilon}{b}\right) \cdot\left(-\frac{b}{\varepsilon}\right) e^{-\frac{b x}{\varepsilon}}\right|_{x_{i-1}} ^{x_{i}}\right. \\
& \left.+\left.\frac{1}{b}\left(\frac{e^{-\rho_{i-1}}}{e^{\rho_{i}-e^{-\rho_{i}}}}\right) \cdot x\right|_{x_{i}} ^{x_{i+1}}+\left.\frac{e^{\alpha_{i}}}{\varepsilon}\left(\frac{1-e^{-\rho_{i}}}{e^{\rho_{i}-e^{-\rho_{i}}}}\right)\left(-\frac{\varepsilon}{b}\right) \cdot\left(-\frac{b}{\varepsilon}\right) e^{-\frac{b x}{\varepsilon}}\right|_{x_{i}} ^{x_{i+1}}\right]
\end{aligned}
$$

Remember that $x_{i+1}=x_{i}+h, x_{i-1}=x_{i}-h$ and $x_{i+1}-x_{i-1}=h$. 
Finally, it can be written as follows

$$
\begin{gathered}
(f-c u) \int_{x_{i-1}}^{x_{i+1}} g_{i} d x=(f-c u)\left(\frac{h}{b}\right)\left(\frac{e^{\rho_{i}}-1}{e^{\rho_{i}}+1}\right) \\
-\varepsilon g_{i}^{\prime}\left(x_{i-1}\right) u_{i-1}+u_{i}+\varepsilon g_{i}^{\prime}\left(x_{i+1}\right) u_{i+1}=(f-c u) \int_{x_{i-1}}^{x_{i+1}} g_{i} d x \\
-\frac{e^{\rho_{i}}-1}{e^{\rho_{i}}-e^{-\rho_{i}}} U_{i-1}+U_{i}-\frac{e^{\rho_{i}}-1}{e^{\rho_{i}}-e^{-\rho_{i}}} U_{i+1}=\left(f_{i}-c_{i} U_{i}\right) \frac{h}{b}\left(\frac{e^{\rho_{i}}+1}{2}\right)
\end{gathered}
$$

Re-arranging above equation and get we get$$
-\frac{e^{\rho_{i}}}{e^{\rho_{i}+1}} U_{i-1}+U_{i}-\frac{1}{e^{\rho_{i}}+1} U_{i+1}=\left(f_{i}-c_{i} U_{i}\right) \frac{h}{b}\left(\frac{e^{\rho_{i}}-1}{e^{\rho_{i}}+1}\right)
$$

This result is exactly same a El-Mistikawy-Werle scheme in [6].

Example 1: In equation (1), if we choose $\mathrm{b}=1, \mathrm{c}=0, \mathrm{u}(0)=0, \mathrm{u}(1)=0$ and $f(x)=2 x$ we get exact solution

$$
u(x)=\frac{1-e^{\frac{x}{\varepsilon}}-x^{2}+x^{2} e^{\frac{1}{\varepsilon}}+2 \varepsilon-2 e^{\frac{x}{\varepsilon}}-2 \varepsilon x+2 \varepsilon x e^{\frac{1}{\varepsilon}}}{-1+e^{\frac{1}{\varepsilon}}}
$$

Above, the solution changes rapidly in the interval $(1-\varepsilon, 1)$. That is, there is an $\varepsilon$ wide boundary layer around $x=1$ as $\varepsilon \rightarrow 0$. This shows that the boundary layer thickness gets thinner as $\varepsilon$ gets smaller. Therefore, it remains important to develop effective algorithms for the numerical solution of such problems.

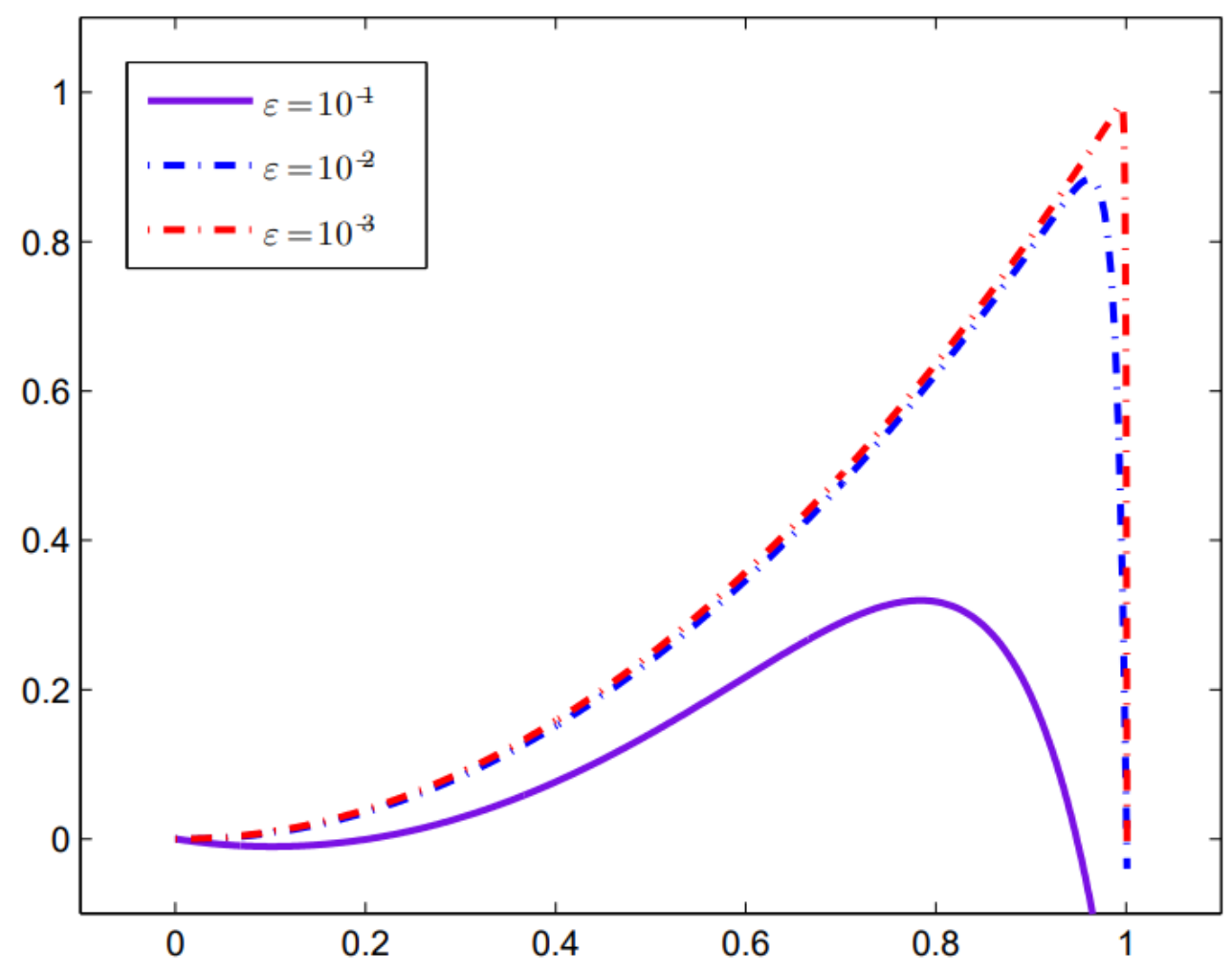

Graphics 1: Exact solution of equation (1) for different $\varepsilon$ values.

\section{CONCLUSION}

In fact, we have obtained Analytical Construction of Uniformly Convergent Method for Convection Diffusion Problem. 


\section{REFERENCES}

[1] Filiz A., Nesliturk, A. I. and Ekici, M., “An $\varepsilon$-uniform numerical method for a singularly-perturbated problem”, XXII Ulusal matematik sempozyumu, Sirince, Izmir, 2009.

[2] Filiz A., " $\varepsilon$-uniform convergence for a boundary value problem", XXIII Ulusal matematik sempozyumu, Erciyes Universitesi, Kayseri, 2010.

[3] Miller, H. J. H., O’Riordan, E. and Shishkin, G. I., Fitted Numerical methods for singular perturbation problems: Error estimates in the maximum norm for linear problems in one and two dimensions, World Scientific, USA, 1996.

[4] Roos, H. G., Ten Ways to Generate the Il'in and Related Schemes", J. of Computational and Appl. Maths., 53 p. $43-$ 59, 1994.

[5] Roos, H. G., Stynes, M. and Tobiska, L., Numerical Methods for Singularly Perturbed Differential Equations, Convection Diffusion and Flow Problems, Springer-Verlag, Berlin Heidelbrg Newyork, 1994.

[6] Roos, H. G., Stynes, M. and Tobiska, L., Robust Numerical Methods for Singularly Perturbed Differential Equations, Convection Diffusion and Flow Problems, 2008.

[7] Stynes, M., Tobiska, L., "A finite difference analysis of a streamline diffusion method on a Shishkin mesh", Numer Algoritms, 18(3-4), pp.337-360, 1998. 